\title{
Late transient acceleration of the universe in string theory on $S^{1} / Z_{2}$
}

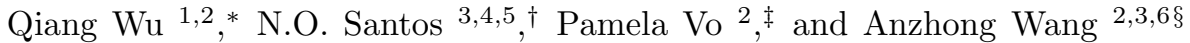 \\ 1 Department of Applied Physics, Zhejiang University of Technology, Hangzhou 310032, China \\ ${ }^{2}$ GCAP-CASPER, Department of Physics, Baylor University, Waco, Texas 76798-7316 \\ ${ }^{3}$ LERMA/CNRS-FRE 2460, Université Pierre et Marie Curie, \\ ERGA, Boîte 142, 4 Place Jussieu, 75005 Paris Cedex 05, France \\ 4 School of Mathematical Sciences, Queen Mary, University of London, London E1 4NS, UK \\ 5 Laboratório Nacional de Computação Científica, 25651-070 Petrópolis RJ, Brazil \\ ${ }^{6}$ Department of Theoretical Physics, the State University of Rio de Janeiro, RJ, Brazil
}

(Dated: December 4, 2018)

\begin{abstract}
Recently, in Gong et al [17] and Wang and Santos [21] it was shown that the effective cosmological constant on each of the two orbifold branes can be easily lowered to its current observational value, by using the large extra dimensions in the framework of both M-Theory and string theory on $S^{1} / Z_{2}$. In this paper, we study the current acceleration of the universe, using the formulas developed in 21]. We first construct explicitly time-dependent solution to the 10-dimensional bulk of the Neveu-Schwarz/Neveu-Schwarz sector, compactified on a 5-dimensional torus. Then, we write down the generalized Friedmann equations on each of the two dynamical branes, and fit the models to the 182 gold supernova Ia data and the BAO parameter from SDSS, using both of our MINUIT and Monte-Carlo Markov Chain (MCMC) codes. With the best fitting values of the parameters involved as initial conditions, we integrate the generalized Friedmann equations numerically and find the future evolution of the universe. We find that it depends on the choice of the radion potentials $V_{4}^{(I)}(I=1,2)$ of the branes. In particular, when choosing them to be the GoldbergerWise potentials, $V_{4}^{(I)}=\lambda_{4}^{(I)}\left(\psi^{2}-v_{I}^{2}\right)^{2}$, we find that the current acceleration of the universe driven by the effective cosmological constant is only temporary. Due to the effects of the potentials, the universe will be finally in its decelerating expansion phase again. We also study the proper distance between the two branes, and find that it remains almost constant during the whole future evolution of the universe in all the models considered.
\end{abstract}

PACS numbers: $03.50 .+\mathrm{h}, 11.10 . \mathrm{Kk}, 98.80 . \mathrm{Cq}, 97.60 .-\mathrm{s}$

\section{INTRODUCTION}

One of the long-standing problems in particle physics and cosmology is the cosmological constant problem: its theoretical expectation values from quantum field theory exceed observational limits by 120 orders of magnitude [1]. Even if such high energies are suppressed by supersymmetry, the electroweak corrections are still 56 orders higher. This problem was further sharpened by recent observations of supernova (SN) Ia, which reveal the striking discovery that our universe has lately been in its accelerated expansion phase [2]. Cross checks from the cosmic microwave background radiation and large scale structure all confirm this unexpected result [3].

In Einstein's theory of gravity, such an expansion can be achieved by a tiny positive cosmological constant. In fact, such a constant is well consistent with all observations carried out so far [4]. Therefore, solving the cosmological constant problem now becomes more urgent than ever before. As a matter of fact, it is exactly because of this that a large number of ambitious projects have been

\footnotetext{
*Electronic address: qiang*wu@baylor.edu

${ }^{\dagger}$ Electronic address: nos@cbpf.br and N.O.Santos@qmul.ac.uk

${ }^{\ddagger}$ Electronic address: pamelaivo@baylor.edu

§Electronic address: anzhong'wang@baylor.edu
}

proposed lately to distinguish the cosmological constant from dynamical dark energy models [5].

Since the problem is intimately related to quantum gravity, its solution is expected to come from quantum gravity, too. At the present, string/M-Theory is our best bet for a consistent quantum theory of gravity, so it is reasonable to ask what string/M-Theory has to say about the cosmological constant. In the string landscape [6], it is expected that there are many different vacua with different local cosmological constants [7]. Using the anthropic principle, one may select the low energy vacuum in which we can exist. However, many theorists still hope to explain the problem without invoking the existence of ourselves in the universe.

Townsend and Wohlfarth [8] considered a timedependent compactification of pure gravity in higher dimensions with hyperbolic internal space to circumvent Gibbons' non-go theorem [9]. Their exact solution exhibits a short period of acceleration. The solution is the zero-flux limit of spacelike branes [10]. If non-zero flux or forms are turned on, a transient acceleration exists for both compact internal hyperbolic and flat spaces [11]. Other accelerating solutions by compactifying more complicated time-dependent internal spaces can be found in 12.

In the same spirit, the cosmological constant problem was also studied in the framework of brane world in 5-dimensional spacetimes [13] and 6-dimensional su- 
pergravity [14]. However, it turned out that in the 5dimensional case hidden fine-tunings are required [15], while in the 6 -dimensional case it is still not clear whether loop corrections can be as small as required [16].

Recently, we [17 studied the cosmological constant problem and late acceleration of the universe in the framework of the Horava-Witten heterotic M-Theory on $S^{1} / Z_{2}$ [18]. In particular, using the Arkani-HamedDimopoulos-Dvali (ADD) mechanism of large extra dimensions [19], we showed explicitly that the effective cosmological constant on each of the two orbifold branes can be easily lowered to its current observational value. Applying it to cosmology, we further found that the domination of the effective cosmological constant is only temporary. Due to the interaction of the bulk and the brane, the universe will be in its decelerating expansion phase again in the future, whereby all problems connected with string cosmology [20] are resolved. Such studies were also generalized to string theory, and found that the ADD mechanism can be used in the same way to solve the cosmological constant problem [21]. Therefore, the ADD mechanism for solving both the cosmological constant problem and the hierarchy problem is a built-in mechanism in the brane world of string/M-Theory.

In this paper, we apply the theory developed in 21] in the framework of string theory on $S^{1} / Z_{2}$ to cosmology, and study the current acceleration of the universe. In particular, in Sec. II we first give a brief review of the theory, and then write down the field equations both in the bulk and on the two orbifold branes. In Sec. III, we present a particular time-dependent solution to these equations, and study its local and global properties. Then, we write down explicitly the generalized Friedmann equations on each of the two branes for any radion potentials $V_{4}^{(I)}(I=1,2)$ of the branes. Depending on the choice of $V_{4}^{(I)}$ 's, the future evolution of the universe is different. We study two different cases. We fit these models to the 182 gold supernova Ia data 22 ] and BAO parameter from SDSS [23], using both of our MINUIT 24] and Monte-Carlo Markov Chain (MCMC) [25] codes. With these best fitting values as the initial condition, we integrate numerically the field equations on the branes to find the future evolution of the universe. In the latter case, we show that the current acceleration of the universe driven by the effective cosmological constant is only temporary. Due to the effects of the potentials, the universe will be in its decelerating expansion phase again. We also study the proper distance between the two orbifold branes, and find that it remains almost constant during the whole future evolution of the universe in all these models. In Sec. IV, we summarize our main results and present some concluding remarks.

Before turning to the next section, we would like to note that Sahni and Shtanov [26] found that transient acceleration of the universe happened in the DGP brane model [27], too.

\section{BRANY COSMOLOGY OF STRING THEORY ON $S^{1} / Z_{2}$}

To begin with, in this section we give a brief review on the cosmological models of orbifold branes developed in 21]. In this paper we shall restrict ourselves directly to the case $D=d=5$. For the case with arbitrary $D$ and $d$, we refer readers to [21].

\section{A. The Model}

For the toroidal compactification of the NeveuSchwarz/Neveu-Schwarz (NS-NS) sector in $(5+5)$ dimensions, $\hat{M}_{10}=M_{5} \times \mathcal{T}_{5}$, where $\mathcal{T}_{5}$ is a 5 -dimensional torus, the action takes the form [28, 29],

$$
\begin{aligned}
\hat{S}_{10}= & -\frac{1}{2 \kappa_{10}^{2}} \int d^{10} x \sqrt{\left|\hat{g}_{10}\right|} e^{-\hat{\Phi}}\left\{\hat{R}_{10}[\hat{g}]\right. \\
& \left.+\hat{g}^{A B}\left(\hat{\nabla}_{A} \hat{\Phi}\right)\left(\hat{\nabla}_{B} \hat{\Phi}\right)-\frac{1}{12} \hat{H}^{2}\right\},
\end{aligned}
$$

where $\hat{\nabla}_{A}$ denotes the covariant derivative with respect to $\hat{g}^{A B}$ with $A, B=0,1, \ldots, 9$, and $\hat{\Phi}$ is the dilaton field. The NS three-form field $\hat{H}_{A B C}$ is defined as

$$
\hat{H}_{A B C}=3 \partial_{[A} \hat{B}_{B C]},
$$

where the square brackets imply total antisymmetrization over all indices. The 10-dimensional spacetimes to be considered are described by the metric,

$$
\begin{aligned}
d \hat{s}_{10}^{2} & =\hat{g}_{A B} d x^{A} d x^{B} \\
& =\tilde{g}_{a b}\left(x^{c}\right) d x^{a} d x^{b}+h_{i j}\left(x^{c}\right) d z^{i} d z^{j},
\end{aligned}
$$

where $\tilde{g}_{a b}$ is the metric on $M_{5}$, parametrized by the coordinates $x^{a}$ with $a, b, c=0,1, \ldots, 4$, and $h_{i j}$ is the metric on the compact space $\mathcal{T}_{5}$ with periodic coordinates $z^{i}$, where $i, j=5,6, \ldots, 9$.

By assuming that all the matter fields are functions of $x^{a}$ only, it can be shown that the effective 5 -dimensional action is given by,

$$
\begin{aligned}
S_{5}= & -\frac{1}{2 \kappa_{5}^{2}} \int d^{5} x \sqrt{\left|\tilde{g}_{5}\right|} e^{-\tilde{\phi}}\left\{\tilde{R}_{5}[\tilde{g}]+\left(\tilde{\nabla}_{a} \tilde{\phi}\right)\left(\tilde{\nabla}^{a} \tilde{\phi}\right)\right. \\
& +\frac{1}{4}\left(\tilde{\nabla}_{a} h^{i j}\right)\left(\tilde{\nabla}^{a} h_{i j}\right)-\frac{1}{12} \tilde{H}_{a b c} \tilde{H}^{a b c} \\
& \left.-\frac{1}{4} h^{i k} h^{j l}\left(\tilde{\nabla}_{a} B_{i j}\right)\left(\tilde{\nabla}^{a} B_{k l}\right)\right\},
\end{aligned}
$$

where

$$
\begin{aligned}
\tilde{\phi} & =\hat{\Phi}-\frac{1}{2} \ln |h|, \\
\kappa_{5}^{2} & \equiv \frac{\kappa_{10}^{2}}{V_{0}},
\end{aligned}
$$

with the 5 -dimensional internal volume given by

$$
V\left(x^{a}\right) \equiv \int d^{5} z \sqrt{|h|}=|h|^{1 / 2} V_{0}
$$


Note that in writing the action (2.4) we had assumed that the flux is block diagonal,

$$
\left(\hat{B}_{C D}\right)=\left(\begin{array}{cc}
\tilde{B}_{a b} & 0 \\
0 & B_{i j}
\end{array}\right) \text {. }
$$

The action (2.4) is usually referred to as written in the string frame. To go to the Einstein frame, we make the following conformal transformations,

$$
\begin{aligned}
g_{a b} & =\Omega^{2} \tilde{g}_{a b}, \\
\Omega^{2} & =\exp \left(-\frac{2}{3} \tilde{\phi}\right), \\
\phi & =\sqrt{\frac{2}{3}} \tilde{\phi} .
\end{aligned}
$$

Then, the action (2.4) takes the form

$$
\begin{aligned}
S_{5}^{(E)}= & -\frac{1}{2 \kappa_{5}^{2}} \int d^{5} x \sqrt{\left|g_{5}\right|}\left\{R_{5}[g]-\frac{1}{2}(\nabla \phi)^{2}\right. \\
& +\frac{1}{4}\left(\nabla_{a} h^{i j}\right)\left(\nabla^{a} h_{i j}\right) \\
& -\frac{1}{12} e^{-\sqrt{\frac{8}{3}} \phi} H_{a b c} H^{a b c} \\
& \left.-\frac{1}{4} h^{i k} h^{j l}\left(\nabla_{a} B_{i j}\right)\left(\nabla^{a} B_{k l}\right)\right\},
\end{aligned}
$$

where $\nabla_{a}$ denotes the covariant derivative with respect to $g_{a b}$. It should be noted that, since the definition of the three-form $\hat{H}_{A B C}$ given by (2.2) is independent of the metric, it is conformally invariant. In particular, we have $H_{a b c}=\tilde{H}_{a b c}$ and $B_{a b}=\tilde{B}_{a b}$. However, we do have

$$
\begin{aligned}
& H^{a b c}=g^{a d} g^{b e} g^{c f} H_{d e f}=\Omega^{-6} \tilde{H}^{a b c}, \\
& H_{a b c} H^{a b c}=\Omega^{-6} \tilde{H}_{a b c} \tilde{H}^{a b c} .
\end{aligned}
$$

Considering the addition of a potential term [29], in the string frame we have

$$
\hat{S}_{10}^{m}=-\int d^{10} x \sqrt{\left|\hat{g}_{10}\right|} V_{10}^{s} .
$$

Then, after the dimensional reduction we find

$$
S_{5, m}=-V_{0} \int d^{5} x \sqrt{\left|\tilde{g}_{5}\right|}|h|^{1 / 2} V_{10}^{s},
$$

where

$$
\tilde{g}_{5}=\exp \left(\sqrt{\frac{50}{3}} \phi\right) g_{5} .
$$

Changed to the Einstein frame, the action (2.13) finally takes the form,

$$
S_{5, m}^{(E)}=-\frac{1}{2 \kappa_{5}^{2}} \int d^{5} x \sqrt{\left|g_{5}\right|} V_{5},
$$

where

$$
V_{5} \equiv 2 \kappa_{5}^{2} V_{0} V_{10}^{s} \exp \left(\frac{5}{\sqrt{6}} \phi\right)|h|^{1 / 2} .
$$

If we further assume that

$$
\begin{aligned}
& h_{i j}=-\exp \left(\sqrt{\frac{2}{5}} \psi\right) \delta_{i j}, \\
& h^{i j}=-\exp \left(-\sqrt{\frac{2}{5}} \psi\right) \delta^{i j},
\end{aligned}
$$

we find that

$$
\begin{aligned}
S_{5}^{(E)}+S_{5, m}^{(E)}= & -\frac{1}{2 \kappa_{5}^{2}} \int d^{5} x \sqrt{\left|g_{5}\right|}\left\{R_{5}[g]\right. \\
& -\frac{1}{2}\left((\nabla \phi)^{2}+(\nabla \psi)^{2}-2 V_{5}\right) \\
& -\frac{1}{4} e^{-\sqrt{\frac{8}{5}} \psi} \delta^{i k} \delta^{j l}\left(\nabla_{a} B_{i j}\right)\left(\nabla^{a} B_{k l}\right) \\
& \left.-\frac{1}{12} e^{-\sqrt{\frac{8}{3}} \phi} H_{a b c} H^{a b c}\right\}
\end{aligned}
$$

where the effective 5-dimensional potential (2.14) now becomes

$$
V_{5} \equiv V_{(5)}^{0} \exp \left(\frac{5}{\sqrt{6}} \phi+\sqrt{\frac{5}{2}} \psi\right)
$$

where $V_{(5)}^{0} \equiv 2 \kappa_{5}^{2} V_{0} V_{10}^{s}$.

To study orbifold branes, we consider the brane actions,

$$
\begin{aligned}
S_{4, m}^{(I)}= & -\int_{M_{4}^{(I)}} \sqrt{\left|g_{4}^{(I)}\right|}\left(\epsilon_{I} V_{4}^{(I)}(\phi, \psi)+g_{s}^{(I)}\right) d^{4} \xi_{(I)} \\
& +\int_{M_{4}^{(I)}} d^{4} \xi_{(I)} \sqrt{\left|g_{4}^{(I)}\right|} \\
& \times \mathcal{L}_{4, m}^{(I)}(\phi, \psi, B, \chi),
\end{aligned}
$$

where $I=1,2, \quad V_{4}^{(I)}(\phi, \psi)$ denotes the potential of the scalar fields $\phi$ and $\psi$, and $\xi_{(I)}^{\mu}$ 's are the intrinsic coordinates of the I-th brane with $\mu, \nu=0,1,2,3$, and $\epsilon_{1}=-\epsilon_{2}=1 . \chi$ denotes collectively the matter fields, and $g_{s}^{(I)}$ is a constant, which is related to the fourdimensional Newtonian constant via the relation given by Eq.(2.39) below. The variation of the total action,

$$
S_{\text {total }}=S_{5}^{(E)}+S_{5, m}^{(E)}+\sum_{I=1}^{2} S_{4, m}^{(I)},
$$

with respect to the metric $g_{a b}$ yields the field equations,

$$
\begin{aligned}
G_{a b}^{(5)}= & \kappa_{5}^{2} T_{a b}^{(5)}+\kappa_{5}^{2} \sum_{I=1}^{2} \mathcal{T}_{\mu \nu}^{(I)} e_{a}^{(I, \mu)} e_{b}^{(I, \nu)} \\
& \times \sqrt{\left|\frac{g_{4}^{(I)}}{g_{5}}\right| \delta\left(\Phi_{I}\right)},
\end{aligned}
$$


where $\delta(x)$ denotes the Dirac delta function normalized in the sense of [30], and the two branes are localized on the surfaces,

$$
\Phi_{I}\left(x^{a}\right)=0 .
$$

The energy-momentum tensors $T_{a b}^{(5)}$ and $\mathcal{T}_{\mu \nu}^{(I)}$ are given by

$$
\begin{aligned}
\kappa_{5}^{2} T_{a b}^{(5)} \equiv & \frac{1}{2}\left[\left(\nabla_{a} \phi\right)\left(\nabla_{b} \phi\right)+\left(\nabla_{a} \psi\right)\left(\nabla_{b} \psi\right)\right. \\
& +\frac{1}{2} e^{-\sqrt{\frac{8}{5}} \psi}\left(\nabla_{a} B^{i j}\right)\left(\nabla_{b} B_{i j}\right) \\
& \left.+\frac{1}{2} e^{\sqrt{\frac{8}{3}} \phi} H_{a c d} H_{b} c d\right] \\
& -\frac{1}{4} g_{a b}\left[(\nabla \phi)^{2}+(\nabla \psi)^{2}-2 V_{5}\right. \\
& \frac{1}{2} e^{-\sqrt{\frac{8}{5}} \psi}\left(\nabla_{c} B^{i j}\right)\left(\nabla^{c} B_{i j}\right) \\
& \left.+\frac{1}{6} e^{\sqrt{\frac{8}{3}} \phi} H_{c d e} H^{c d e}\right] \\
\mathcal{T}_{\mu \nu}^{(I)} \equiv & \tau_{\mu \nu}^{(I)}+\left(g_{s}^{(I)}+\tau_{(\phi, \psi)}^{(I)}\right) g_{\mu \nu}^{(I)} \\
\tau_{\mu \nu}^{(I)} \equiv & 2 \frac{\delta \mathcal{L}_{4, m}^{(I)}}{\delta g^{(I) \mu \nu}}-g_{\mu \nu}^{(I)} \mathcal{L}_{4, m}^{(I)}
\end{aligned}
$$

where $B^{i j} \equiv \delta^{i k} \delta^{j l} B_{k l}$,

$$
\begin{aligned}
\tau_{(\phi, \psi)}^{(I)} & \equiv \epsilon_{I} V_{4}^{(I)}(\phi, \psi), \\
e_{(\mu)}^{(I) a} & \equiv \frac{\partial x^{a}}{\partial \xi_{(I)}^{\mu}}, \\
e_{a}^{(I, \mu)} & \equiv g_{a b} g^{(I) \mu \nu} e_{(\nu)}^{(I) b},
\end{aligned}
$$

and $g_{\mu \nu}^{(I)}$ is the reduced metric on the I-th brane, defined as

$$
\left.g_{\mu \nu}^{(I)} \equiv g_{a b} e_{(\mu)}^{(I) a} e_{(\nu)}^{(I) b}\right|_{M_{4}^{(I)}} .
$$

Variation of the total action Eq. 2.21) with respect to $\phi, \psi$ and $B$, respectively, yields the following equations of the matter fields,

$$
\begin{aligned}
\square \phi= & -\frac{\partial V_{5}}{\partial \phi}-\frac{1}{12} \sqrt{\frac{8}{3}} e^{-\sqrt{\frac{8}{3}} \phi} H_{a b c} H^{a b c} \\
& -2 \kappa_{5}^{2} \sum_{I=1}^{2}\left(\epsilon_{I} \frac{\partial V_{4}^{(I)}}{\partial \phi}+\sigma_{\phi}^{(I)}\right) \\
& \times \sqrt{\left|\frac{g_{4}^{(I)}}{g_{5}}\right| \delta\left(\Phi_{I}\right)} \\
\square \psi= & -\frac{\partial V_{5}}{\partial \psi}-\sqrt{\frac{1}{10}} e^{-\sqrt{\frac{8}{5}} \psi}\left(\nabla_{a} B^{i j}\right)\left(\nabla^{a} B_{i j}\right) \\
& -2 \kappa_{5}^{2} \sum_{I=1}^{2}\left(\epsilon_{I} \frac{\partial V_{4}^{(I)}}{\partial \psi}+\sigma_{\psi}^{(I)}\right)
\end{aligned}
$$

$$
\begin{aligned}
& \times \sqrt{\left|\frac{g_{4}^{(I)}}{g_{5}}\right|} \delta\left(\Phi_{I}\right), \\
\square B_{i j}= & \sqrt{\frac{8}{5}}\left(\nabla_{a} \psi\right)\left(\nabla^{a} B_{i j}\right) \\
& -\sum_{I=1}^{2} \Psi_{i j}^{(I)} \sqrt{\left|\frac{g_{4}^{(I)}}{g_{5}}\right|} \delta\left(\Phi_{I}\right), \\
\nabla^{c} H_{c a b}= & \sqrt{\frac{8}{3}} H_{c a b} \nabla^{c} \phi \\
& -\sum_{I=1}^{2} \Phi_{a b}^{(I)} \sqrt{\left|\frac{g_{4}^{(I)}}{g_{5}}\right|} \delta\left(\Phi_{I}\right),
\end{aligned}
$$

where $\square \equiv g^{a b} \nabla_{a} \nabla_{b}$, and

$$
\begin{aligned}
\sigma_{\phi}^{(I)} & \equiv-\frac{\delta \mathcal{L}_{4, m}^{(I)}}{\delta \phi}, \\
\sigma_{\psi}^{(I)} & \equiv-\frac{\delta \mathcal{L}_{4, m}^{(I)}}{\delta \psi}, \\
\Psi_{i j}^{(I)} & \equiv-4 \kappa_{5}^{2} e^{\sqrt{\frac{8}{5}}} \psi \frac{\delta \mathcal{L}_{4, m}^{(I)}}{\delta B^{i j}}, \\
\Phi_{a b}^{(I)} & \equiv-4 \kappa_{5}^{2} e^{\sqrt{\frac{8}{3}}} \frac{\delta \mathcal{L}_{4, m}^{(I)}}{\delta B^{a b}} .
\end{aligned}
$$

To write down the field equations on the branes, one can first express the delta function part of $G_{a b}^{(5)}$ in terms of the discontinuities of the first derivatives of the metric coefficients, and then equal the delta function parts of the two sides of Eq.(2.22), as shown systematically in [31]. The other way is to use the Gauss-Codacci equations to write the (4)-dimensional Einstein tensor as [32],

$$
G_{\mu \nu}^{(4)}=\mathcal{G}_{\mu \nu}^{(5)}+E_{\mu \nu}^{(5)}+\mathcal{F}_{\mu \nu}^{(4)},
$$

where

$$
\begin{aligned}
\mathcal{G}_{\mu \nu}^{(5)} \equiv & \frac{2}{3}\left\{G_{a b}^{(5)} e_{(\mu)}^{a} e_{(\nu)}^{b}\right. \\
& \left.-\left[G_{a b} n^{a} n^{b}+\frac{1}{4} G^{(5)}\right] g_{\mu \nu}\right\}, \\
E_{\mu \nu}^{(5)} \equiv & C_{a b c d}^{(5)} n^{a} e_{(\mu)}^{b} n^{c} e_{(\nu)}^{d}, \\
\mathcal{F}_{\mu \nu}^{(4)} \equiv & K_{\mu \lambda} K_{\nu}^{\lambda}-K K_{\mu \nu} \\
& -\frac{1}{2} g_{\mu \nu}\left(K_{\alpha \beta} K^{\alpha \beta}-K^{2}\right),
\end{aligned}
$$

where $n^{a}$ denotes the normal vector to the brane, $G^{(5)} \equiv$ $g^{a b} G_{a b}^{(5)}$, and $C_{a b c d}^{(5)}$ the Weyl tensor. The extrinsic curvature $K_{\mu \nu}$ is defined as

$$
K_{\mu \nu} \equiv e_{(\mu)}^{a} e_{(\nu)}^{b} \nabla_{a} n_{b} .
$$

A crucial step of this approach is the Lanczos equations [33],

$$
\left[K_{\mu \nu}^{(I)}\right]^{-}-g_{\mu \nu}^{(I)}\left[K^{(I)}\right]^{-}=-\kappa_{5}^{2} \mathcal{T}_{\mu \nu}^{(I)}
$$


where

$$
\begin{aligned}
& {\left[K_{\mu \nu}^{(I)}\right]^{-} \equiv \lim _{\Phi_{I} \rightarrow 0^{+}} K_{\mu \nu}^{(I)+}-\lim _{\Phi_{I} \rightarrow 0^{-}} K_{\mu \nu}^{(I)-}} \\
& {\left[K^{(I)}\right]^{-} \equiv g^{(I) \mu \nu}\left[K_{\mu \nu}^{(I)}\right]^{-}}
\end{aligned}
$$

Assuming that the branes have $Z_{2}$ symmetry, we can express the intrinsic curvatures $K_{\mu \nu}^{(I)}$ in terms of the effective energy-momentum tensor $\mathcal{T}_{\mu \nu}^{(I)}$ through the Lanczos equations (2.35). Then, we find that $G_{\mu \nu}^{(4)}$ given by Eq. (2.32) can be cast in the form,

$$
\begin{aligned}
G_{\mu \nu}^{(4)}= & \mathcal{G}_{\mu \nu}^{(5)}+E_{\mu \nu}^{(5)}+\mathcal{E}_{\mu \nu}^{(4)} \\
& +\kappa_{4}^{2} \tau_{\mu \nu}+\Lambda g_{\mu \nu}+\kappa_{5}^{4} \pi_{\mu \nu}
\end{aligned}
$$

where

$$
\begin{aligned}
\pi_{\mu \nu} \equiv & \frac{1}{4}\left\{\tau_{\mu \lambda} \tau_{\nu}^{\lambda}-\frac{1}{3} \tau \tau_{\mu \nu}\right. \\
& \left.-\frac{1}{2} g_{\mu \nu}\left(\tau^{\alpha \beta} \tau_{\alpha \beta}-\frac{1}{3} \tau^{2}\right)\right\}, \\
\mathcal{E}_{\mu \nu}^{(4)} \equiv & \frac{\kappa_{5}^{4}}{6} \tau_{(\phi, \psi)}\left[\tau_{\mu \nu}+\left(g_{s}+\frac{1}{2} \tau_{(\phi, \psi)}\right) g_{\mu \nu}\right],
\end{aligned}
$$

and

$$
\begin{aligned}
\kappa_{4}^{2} & =\frac{1}{6} g_{s} \kappa_{5}^{4}, \\
\Lambda & =\frac{1}{12} g_{s}^{2} \kappa_{5}^{4} .
\end{aligned}
$$

For a perfect fluid,

$$
\tau_{\mu \nu}=(\rho+p) u_{\mu} u_{\nu}-p g_{\mu \nu}
$$

where $u_{\mu}$ is the four-velocity of the fluid, we find that

$$
\pi_{\mu \nu}=\frac{\rho}{6}\left[(\rho+p) u_{\mu} u_{\nu}-\left(p+\frac{1}{2} \rho\right) g_{\mu \nu}\right] .
$$

Note that in writing Eqs.2.37)-2.41), without causing any confusion, we had dropped the super indices $(I)$.

In the rest of this paper, we shall turn off the flux, i.e., $\hat{B}_{C D}=0$, which is consistent with the field equations, provided that $\Psi_{i j}^{(I)}=0$ and $\Phi_{a b}^{(I)}=0$.

\section{B. The General Metric of the Five-Dimensional Spacetimes}

Since we shall apply such spacetimes to cosmology, let us first consider the embedding of a 3-dimensional spatial space that is homogeneous, isotropic, and independent of time. It is not difficult to show that such a space must have a constant curvature and its metric takes the form [34],

$$
d \Sigma_{k}^{2}=\frac{d r^{2}}{1-k r^{2}}+r^{2}\left(d \theta^{2}+\sin ^{2} \theta d \phi^{2}\right)
$$

where the constant $k$ represents the curvature of the 3space, and can be positive, negative or zero. Without loss of generality, we shall choose coordinates such that $k=0, \pm 1$. Then, one can see that the most general metric for the five-dimensional spacetime must take the form,

$$
d s_{5}^{2}=g_{a b} d x^{a} d x^{b}=g_{M N} d x^{M} d x^{N}-e^{2 \omega\left(x^{N}\right)} d \Sigma_{k}^{2},
$$

where $M, N=0,1$. Clearly, the metric (2.43) is invariant under the coordinate transformations,

$$
x^{\prime N}=f^{N}\left(x^{M}\right) .
$$

Using these two degrees of freedom, without loss of generality, we can always set

$$
g_{00}=g_{11}, \quad g_{01}=0,
$$

so that the five-dimensional metric finally takes the form,

$$
d s_{5}^{2}=e^{2 \sigma(t, y)}\left(d t^{2}-d y^{2}\right)-e^{2 \omega(t, y)} d \Sigma_{k}^{2} .
$$

It should be noted that metric (2.46) is still subjected to the gauge freedom,

$$
t=f\left(t^{\prime}+y^{\prime}\right)+g\left(t^{\prime}-y^{\prime}\right), \quad y=f\left(t^{\prime}+y^{\prime}\right)-g\left(t^{\prime}-y^{\prime}\right),
$$

where $f\left(t^{\prime}+y^{\prime}\right)$ and $g\left(t^{\prime}-y^{\prime}\right)$ are arbitrary functions of their indicated arguments.

It is also interesting to note that in 31 a different gauge was used. Instead of setting $g_{00}=g_{11}$ it was chosen that the two branes are comoving with the coordinates, so that they are located on two fixed hypersurfaces $y=$ $0, y_{c}$. For details, see [31].

\section{The Field Equations Outside the Two Orbifold Branes}

The non-vanishing components of the Ricci tensor outside of the two branes are given by

$$
\begin{aligned}
R_{t t}^{(5)}= & \sigma_{, y y}+3 \sigma_{, y} \omega_{, y}-\left[\sigma_{, t t}+3 \omega_{, t t}+3 \omega_{, t}\left(\omega_{, t}-\sigma_{, t}\right)\right] \\
R_{t y}^{(5)}= & -3\left[\omega_{, t y}+\omega_{, t} \omega_{, y}-\left(\sigma_{, t} \omega_{, y}+\sigma_{, y} \omega_{, t}\right)\right] \\
R_{y y}^{(5)}= & \sigma_{, t t}+3 \sigma_{, t} \omega_{, t}-\left[\sigma_{, y y}+3 \omega_{, y y}+3 \omega_{, y}\left(\omega_{, y}-\sigma_{, y}\right)\right] \\
R_{m n}^{(5)}= & -e^{-2 \sigma} g_{m n}\left\{\omega_{, t t}+3 \omega_{, t}^{2}-\left(\omega_{, y y}+3 \omega_{, y}^{2}\right)\right. \\
& \left.+2 k e^{2(\sigma-\omega)}\right\}
\end{aligned}
$$

where now $m, n=r, \theta, \varphi, \sigma_{, t} \equiv \partial \sigma / \partial t$ and so on. Then, it can be shown that outside of the two branes the field equations have four independent components, which can be cast into the form,

$$
\begin{aligned}
& \omega_{, t t}+\omega_{, t}\left(\omega_{, t}-2 \sigma_{, t}\right)+\omega_{, y y}+\omega_{, y}\left(\omega_{, y}-2 \sigma_{, y}\right) \\
&=-\frac{1}{6}\left[\left(\phi_{, t}{ }^{2}+\phi_{, y}{ }^{2}\right)+\left(\psi_{, t}{ }^{2}+\psi_{, y}{ }^{2}\right)\right],
\end{aligned}
$$




$$
\begin{gathered}
2 \sigma_{, t t}+\omega_{, t t}-3 \omega_{, t^{2}}-\left(2 \sigma_{, y y}+\omega_{, y y}-3 \omega_{, y}^{2}\right)-4 k e^{2(\sigma-\omega)} \\
=-\frac{1}{2}\left[\left(\phi_{, t}{ }^{2}-\phi_{, y}{ }^{2}\right)+\left(\psi_{, t}{ }^{2}-\psi_{, y}{ }^{2}\right)\right], \\
\begin{aligned}
\omega_{, t y}+\omega_{, t} \omega_{, y} & -\left(\sigma_{, t} \omega_{, y}+\sigma_{, y} \omega_{, t}\right) \\
& =-\frac{1}{6}\left(\phi_{, t} \phi_{, y}+\psi_{, t} \psi, y\right)
\end{aligned} \\
\omega_{, t t}+3 \omega_{, t}{ }^{2}-\left(\omega_{, y y}+3 \omega_{, y}{ }^{2}\right)+2 k e^{2(\sigma-\omega)} \\
=\frac{1}{3} e^{2 \sigma} V_{5},
\end{gathered}
$$

where $V_{5}$ is given by Eq.(2.19). On the other hand, the Klein-Gordon equations (2.27) and (2.28) outside the two branes take the form,

$$
\begin{gathered}
\phi_{, t t}+3 \phi_{, t} \omega_{, t}-\left(\phi_{, y y}+3 \phi_{, y} \omega_{, y}\right) \\
=-\frac{5}{\sqrt{6}} V_{5} e^{2 \sigma}, \\
\psi_{, t t}+3 \psi_{, t} \omega_{, t}-\left(\psi_{, y y}+3 \psi_{, y} \omega_{, y}\right) \\
=-\sqrt{\frac{5}{2}} V_{5} e^{2 \sigma} .
\end{gathered}
$$

\section{The Field Equations on the Two Orbifold Branes}

Eqs. (2.48) - (2.54) are the field equations that are valid in between the two orbifold branes, $y_{2}\left(t_{2}\right)<y<y_{1}\left(t_{1}\right)$, where $y=y_{I}\left(t_{I}\right)$ denote the locations of the two branes. The proper distance between the two branes is given by,

$$
\mathcal{D} \equiv \int_{y_{2}}^{y_{1}} \sqrt{-g_{y y}} d y .
$$

On each of the two branes, the metric reduces to

$$
\left.d s_{5}^{2}\right|_{M_{4}^{(I)}}=g_{\mu \nu}^{(I)} d \xi_{(I)}^{\mu} d \xi_{(I)}^{\nu}=d \tau_{I}^{2}-a^{2}\left(\tau_{I}\right) d \Sigma_{k}^{2},
$$

where $\xi_{(I)}^{\mu} \equiv\left\{\tau_{I}, r, \theta, \varphi\right\}$, and $\tau_{I}$ denotes the proper time of the I-th brane, defined by

$$
\begin{aligned}
d \tau_{I} & =e^{\sigma} \sqrt{1-\left(\frac{\dot{y}_{I}}{\dot{t}_{I}}\right)^{2}} d t_{I}, \\
a\left(\tau_{I}\right) & \equiv \exp \left\{\omega\left[t_{I}\left(\tau_{I}\right), y_{I}\left(\tau_{I}\right)\right]\right\},
\end{aligned}
$$

with $\dot{y}_{I} \equiv d y_{I} / d \tau_{I}$, etc. For the sake of simplicity and without causing any confusion, from now on we shall drop all the indices "I", unless some specific attention is needed. Then, the normal vector $n_{a}$ and the tangential vectors $e_{(\mu)}^{a}$ are given, respectively, by

$$
\begin{aligned}
n_{a} & =\epsilon e^{2 \sigma}\left(-\dot{y} \delta_{a}^{t}+\dot{t} \delta_{a}^{y}\right) \\
n^{a} & =-\epsilon\left(\dot{y} \delta_{t}^{a}+\dot{t} \delta_{y}^{a}\right) \\
e_{(\tau)}^{a} & =\dot{t} \delta_{t}^{a}+\dot{y} \delta_{y}^{a}, \quad e_{(r)}^{a}=\delta_{r}^{a} \\
e_{(\theta)}^{a} & =\delta_{\theta}^{a}, \quad e_{(\varphi)}^{a}=\delta_{\varphi}^{a},
\end{aligned}
$$

where $\epsilon= \pm 1$. When $\epsilon=+1$, the normal vector $n^{a}$ points toward the increasing direction of $y$, and when $\epsilon=-1$, it points toward the decreasing direction of $y$. Then, the four-dimensional field equations on each of the two branes take the form,

$$
\begin{aligned}
H^{2}+ & \frac{k}{a^{2}}=\frac{8 \pi G}{3}\left(\rho+\tau_{(\phi, \psi)}\right)+\frac{1}{3} \Lambda+\frac{1}{3} \mathcal{G}_{\tau}^{(5)}+E^{(5)} \\
& +\frac{2 \pi G}{3 \rho_{\Lambda}}\left(\rho+\tau_{(\phi, \psi)}\right)^{2} \\
\frac{\ddot{a}}{a}= & -\frac{4 \pi G}{3}\left(\rho+3 p-2 \tau_{(\phi, \psi)}\right)+\frac{1}{3} \Lambda \\
& -E^{(5)}-\frac{1}{6}\left(\mathcal{G}_{\tau}^{(5)}+3 \mathcal{G}_{\theta}^{(5)}\right)-\frac{2 \pi G}{3 \rho_{\Lambda}}[\rho(2 \rho+3 p) \\
& \left.+\left(\rho+3 p-\tau_{(\phi, \psi)}\right) \tau_{(\phi, \psi)}\right],
\end{aligned}
$$

where $H \equiv \dot{a} / a, \rho_{\Lambda} \equiv \Lambda_{4} /\left(8 \pi G_{4}\right)$, and

$$
\begin{aligned}
\mathcal{G}_{\tau}^{(5)} \equiv & \frac{1}{3} e^{-2 \sigma}\left[\left(\phi_{, t}{ }^{2}+\psi_{, t}{ }^{2}\right)-\left(\phi_{, y}{ }^{2}+\psi_{, y}{ }^{2}\right)\right] \\
& -\frac{1}{24}\left\{5\left[(\nabla \phi)^{2}+(\nabla \psi)^{2}\right]-6 V_{5}\right\}, \\
\mathcal{G}_{\theta}^{(5)} \equiv & \frac{1}{24}\left\{8\left(\phi_{, n}{ }^{2}+\psi_{, n}{ }^{2}\right)-6 V_{5}\right. \\
& \left.+5\left[(\nabla \phi)^{2}+(\nabla \psi)^{2}\right]\right\}, \\
E^{(5)} \equiv & \frac{1}{6} e^{-2 \sigma}\left[\left(\sigma_{, t t}-\omega_{, t t}\right)-\left(\sigma_{, y y}-\omega_{, y y}\right)\right. \\
& \left.+k e^{2(\sigma-\omega)}\right],
\end{aligned}
$$

with $\phi_{, n} \equiv n^{a} \nabla_{a} \phi$. If the typical size of the extra dimensions is $R$, then it can be shown that

$$
\rho_{\Lambda}=\frac{\Lambda_{4}}{8 \pi G_{4}}=3\left(\frac{R}{l_{p l}}\right)^{10}\left(\frac{M_{10}}{M_{p l}}\right)^{16} M_{p l}^{4}
$$

where $M_{p l}$ and $l_{p l}$ denote the Planck mass and length, respectively. If $M_{10}$ is in the order of $\mathrm{TeV}$ [35], we find that, in order to have $\rho_{\Lambda}$ be in the order of its current observations value $\rho_{\Lambda} \simeq 10^{-47} \mathrm{GeV}^{4}$, the typical size of the extra dimensions should be $R \simeq 10^{-22} \mathrm{~m}$, which is well below the current experimental limit of the extra dimensions [36].

\section{A PARTICULAR MODEL}

In this section, we consider a specific solution of the five-dimensional bulk and the corresponding Friedmann equations on the orbifold branes.

\section{A. Exact Solutions in the Bulk}

It can be shown that the following solution satisfies the field equations in the bulk,

$$
\sigma(t)=\frac{1}{9} \ln (t)+\frac{1}{2} \ln \left(\frac{7}{6}\right)
$$




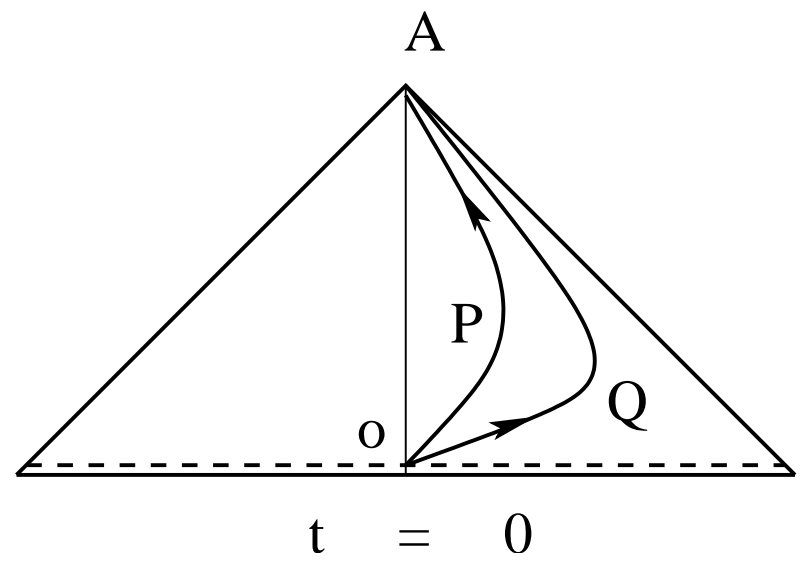

FIG. 1: The Penrose diagram for the metric given by Eq. 3.3. in the text, where the spacetime is singular at $t=0$. The curves $O P A$ and $O Q A$ describes the history of the two orbifold branes located on the surfaces $y=y_{I}\left(\tau_{I}\right)$ with $I=1,2$. The bulk is the region between these two lines.

$$
\begin{aligned}
& \omega(t)=\frac{10}{9} \ln (t), \\
& \phi(t)=-\frac{5}{18} \sqrt{6} \ln (t)+\phi_{0}, \\
& \psi(t)=-\frac{\sqrt{10}}{6} \ln (t)+\psi_{0},
\end{aligned}
$$

for $k=-1$, where

$$
\phi_{0}=\frac{\sqrt{6}}{5}\left\{\ln \left(\frac{2}{3 V_{(5)}^{0}}\right)-\sqrt{\frac{5}{2}} \psi_{0}\right\},
$$

with $\psi_{0}$ being an arbitrary constant. Then, the corresponding 5-dimensional metric takes the form,

$$
d s_{5}^{2}=\left(\frac{7}{6}\right) t^{2 / 9}\left(d t^{2}-d y^{2}\right)-t^{20 / 9} d \Sigma_{-1}^{2} .
$$

Clearly, the spacetime is singular at $t=0$ where all the four spatial dimensions collapse into a point singularity, a big bang like. This can be seen more clearly from the expression,

$$
\psi_{, a} \psi^{, a}=\frac{3}{5} \phi_{, a} \phi^{, a}=\frac{5}{21} t^{-20 / 9} .
$$

The corresponding Penrose diagram is given by Fig. 1 .

Lifting the solution to the 10-dimensional superstring spacetime, we find that in the string frame the metric (2.3) takes the form,

$$
\begin{aligned}
d \hat{s}_{10}^{2}= & \hat{g}_{A B} d x^{A} d x^{B} \\
= & e^{\sqrt{\frac{2}{3}} \phi_{0}}\left\{\left(\frac{7}{6}\right) t^{-1 / 3}\left(d t^{2}-d y^{2}\right)-t^{5 / 3} d \Sigma_{-1}^{2}\right\} \\
& -e^{\sqrt{\frac{2}{5}} \psi_{0}} t^{-1 / 3} \delta_{i j} d z^{i} d z^{j} .
\end{aligned}
$$

The corresponding dilaton field is given by

$$
\hat{\Phi}=-\frac{5}{3} \ln (t)+\hat{\Phi}_{0},
$$

where $\hat{\Phi}_{0} \equiv \sqrt{3 / 2} \phi_{0}+\sqrt{5 / 2} \psi_{0}$, from which we find

$$
\hat{\Phi}_{, A} \hat{\Phi}^{, A}=\frac{50}{21} e^{-\sqrt{\frac{2}{3}} \phi_{0}} t^{-5 / 3} .
$$

Clearly, it is also singular at $t=0$, but with a weaker strength in comparing to that of the five-dimensional spacetime given by Eq.(2.19). A critical difference is that in the string frame the proper distance along the $y$-direction becomes decreasing as $t$ increases, in contrast to that in the Einstein frame, as can be seen clearly from Eqs. (3.3) and (3.5).

\section{B. Generalized Friedmann Equations on The Branes}

On the other hand, from Eq.(2.61) we find that

$$
\begin{aligned}
& E^{(5)}=-\frac{1}{42 a^{2}}, \quad \mathcal{G}_{\tau}^{(5)}=\frac{31}{126 a^{2}}, \\
& \mathcal{G}_{\theta}^{(5)}=\frac{20}{81 a^{9 / 5}} \dot{y}^{2}-\frac{13}{378 a^{2}},
\end{aligned}
$$

where now $a(\tau)=t^{10 / 9}(\tau)$, and $\dot{y}$ is given by

$$
\dot{y}=\epsilon_{y} a^{9 / 10}\left[\left(\frac{9}{10}\right)^{2} H^{2}-\frac{6}{7 a^{2}}\right]^{1 / 2},
$$

with $\epsilon_{y}= \pm 1$. Inserting Eqs.(3.8) and (3.9) into Eqs.(2.59) and (2.60), we find that

$$
\begin{aligned}
H^{2}= & \frac{8 \pi G}{3}\left(\rho+\tau_{(\phi, \psi)}+\rho_{\Lambda}\right)+\frac{200}{189 a^{2}} \\
& +\frac{2 \pi G}{3 \rho_{\Lambda}}\left(\rho+\tau_{(\phi, \psi)}\right)^{2}, \\
\frac{\ddot{a}}{a}= & \frac{4 \pi G}{5}\left(3 \rho_{\Lambda}+3 \tau_{(\phi, \psi)}-2 \rho-5 p\right) \\
& -\frac{2 \pi G}{3 \rho_{\Lambda}}\left[\frac{1}{10}\left(\rho+\tau_{(\phi, \psi)}\right)^{2}+\rho(2 \rho+3 p)\right. \\
& \left.+\left(\rho+3 p-\tau_{(\phi, \psi)}\right) \tau_{(\phi, \psi)}\right] .
\end{aligned}
$$

It is remarkable to note that these two equations do not depend on both $\epsilon$ defined in Eq.(2.58) and $\epsilon_{y}$ defined in Eq.(3.9). Combining Eqs.(3.10) and (3.11), we obtain

$$
\begin{aligned}
\left(\dot{\rho}+\dot{\tau}_{(\phi, \psi)}\right)+3 H(\rho+p)= & -\frac{H}{20 \Delta}\left[4\left(\rho+\rho_{\Lambda}+\tau_{(\phi, \psi)}\right)\right. \\
& \left.+\frac{\left(\rho+\tau_{(\phi, \psi)}\right)^{2}}{\rho_{\Lambda}}\right], \quad(3.12)
\end{aligned}
$$

where

$$
\Delta \equiv 1+\frac{1}{2 \rho_{\Lambda}}\left(\rho+\tau_{(\phi, \psi)}\right)
$$

Eq. (3.12) shows clearly the interaction among the matter fields confined on the branes and the bulk. This can also be seen from Eq.(3.8). 


\section{Current Acceleration of the Universe}

To study current acceleration of the universe, we first set

$$
p=0,
$$

and then introduce the quantities,

$$
\begin{aligned}
\Omega_{m} & =\frac{\rho_{m}}{\rho_{c r}}, \quad \Omega_{\tau}=\frac{\tau_{(\phi, \psi)}}{\rho_{c r}}, \quad \Omega_{\Lambda}=\frac{\rho_{\Lambda}}{\rho_{c r}}, \\
\Omega_{k} & =\frac{200}{189 H_{0}^{2} a^{2}}=\frac{\Omega_{k}^{(0)}}{a^{2}},
\end{aligned}
$$

where $\rho_{c r} \equiv 3 H_{0}^{2} / 8 \pi G$. It should be noted the slight difference between $\Omega_{k}$ defined here and the one normally used, $\Omega_{k}=-k /\left(H_{0}^{2} a^{2}\right)$. Then, Eqs.(3.10), (3.12) and (3.9) can be written as

$$
\begin{aligned}
E^{2}= & \Omega_{\Lambda}+\Omega_{t}+\Omega_{k}+\frac{\Omega_{t}{ }^{2}}{4 \Omega_{\Lambda}}, \\
\Omega_{t}^{*}= & -\frac{E}{\Delta}\left\{\frac{1}{5}\left(\Omega_{\Lambda}+16 \Omega_{t}-15 \Omega_{\tau}\right)\right. \\
& \left.+\frac{\Omega_{t}}{20 \Omega_{\Lambda}}\left(31 \Omega_{t}-30 \Omega_{\tau}\right)\right\}, \\
y^{*}= & \epsilon_{y}\left(\frac{9}{10}\right)\left(\frac{\Omega_{k}^{(0)}}{\Omega_{k}}\right)^{9 / 20} \\
& \times \sqrt{\Omega_{\Lambda}+\Omega_{t}+\frac{\Omega_{t}{ }^{2}}{4 \Omega_{\Lambda}}},
\end{aligned}
$$

where $E \equiv H / H_{0}, y^{*} \equiv d y / d\left(H_{0} \tau\right)$, and

$$
\Omega_{t}=\Omega_{m}+\Omega_{\tau}
$$

with the constraint,

$$
1=\Omega_{k}^{(0)}+\Omega_{\Lambda}+\Omega_{t}^{(0)}+\frac{\Omega_{t}^{(0)}{ }^{2}}{4 \Omega_{\Lambda}}
$$

where $\Omega_{N}^{(0)}$ 's denote their current values. On the other hand, in terms of $\Omega$ 's, we find

$$
\begin{aligned}
\frac{a^{* *}}{a}= & \frac{3}{10}\left(3 \Omega_{\Lambda}-2 \Omega_{t}+5 \Omega_{\tau}\right) \\
& +\frac{3 \Omega_{t}}{40 \Omega_{\Lambda}}\left(7 \Omega_{t}-10 \Omega_{\tau}\right) .
\end{aligned}
$$

To study Eqs.(3.16)-(3.18) and (3.21) further, we need to specify $\Omega_{\tau}$. In the following, we shall consider two different cases.

$$
\text { 1. } V_{4}^{(I)}=V_{(4)}^{0} \exp \left\{\frac{n}{2}\left(\frac{5}{\sqrt{6}} \phi+\sqrt{\frac{5}{2}} \psi\right)\right\}
$$

If we choose the potential $V_{4}^{(I)}(\phi, \psi)$ on each of the two branes as [cf. Eq.(2.19)],

$$
V_{4}^{(I)}=V_{(4)}^{0} \exp \left\{\frac{n}{2}\left(\frac{5}{\sqrt{6}} \phi+\sqrt{\frac{5}{2}} \psi\right)\right\},
$$

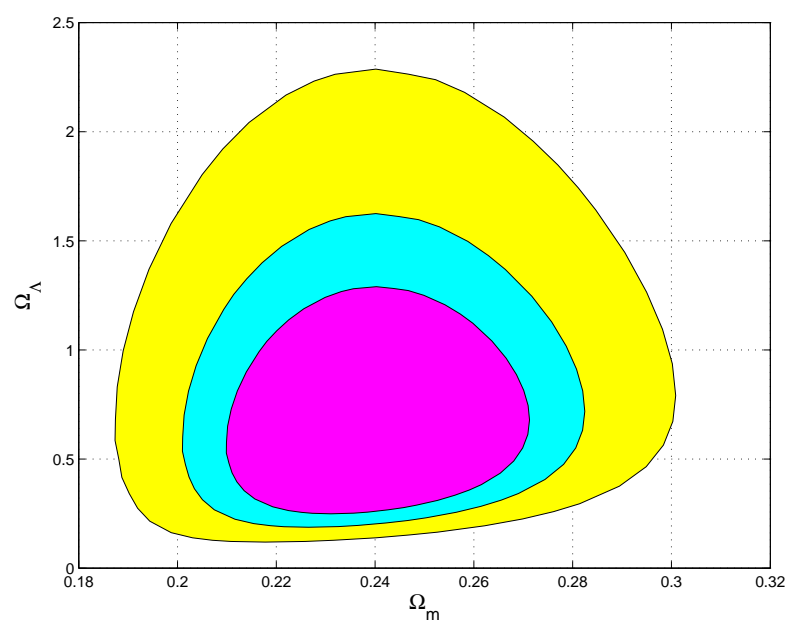

FIG. 2: The marginalized contour of $\Omega_{m}-\Omega_{\Lambda}$ for the potential given by Eq. (3.22) with $n=1$.

where $V_{(4)}^{0}$ and $n$ are arbitrary constants, we find that

$$
\Omega_{\tau}=\epsilon_{I} \frac{V_{(4)}^{0}}{\rho_{c r}}\left(\frac{2}{3 V_{(5)}^{0}}\right)^{n / 2} \frac{1}{a^{n}} \equiv \frac{\Omega_{\tau}^{(0)}}{a^{n}} .
$$

Then, our fitting parameters in this case can be chosen as

$$
\left\{\Omega_{\Lambda}, \Omega_{m}^{(0)}, \Omega_{k}^{(0)}\right\}
$$

for any given $n$.

Fitting the above model to the 182 gold supernova Ia data 22] and the BAO parameter from SDSS [23], by using our numerical code [24], based on the publicly available MINUIT program of CERN, we find that, for $n=1$, the best fitting is $\Omega_{m}=0.24 \pm_{0.03}^{0.03}, \Omega_{\Lambda}=0.76 \pm_{0.27}^{0.37}$, and $\Omega_{k}=0.00 \pm{ }_{0.00}^{0.05}$ with $\chi^{2}=172.4$. Figs. $2-4$ show the marginalized contours of the $\Omega$ 's, from which we can see that the effect of the interaction between the bulk and the brane is negligible, and the later evolution of the universe follows more or less the same pattern as that of the $\Lambda$ CDM model in the Einstein theory of gravity.

For $n=3.5$, we find that the best fitting is $\Omega_{m}=$ $0.27 \pm_{0.03}^{0.03}, \Omega_{\Lambda}=0.58 \pm_{0.12}^{0.11}$, and $\Omega_{k}=0.00 \pm_{0.00}^{0.06}$ with $\chi^{2}=164.2$. Figs. $5-7$ show the marginalized contours of the $\Omega$ 's.

The above shows clearly that the case with $n=3.5$ is observationally more favorable than that of $n=1$. We have also fitted the data with various values of $n$, and found that the best fitting value of $n$ is about $n=3.5$.

With the above best fitting values of the $\Omega$ 's and $n$ as initial conditions, the future evolution of the universe is shown in Figs. 8 and 9, from which we can see that all of them, except for $\Omega_{\Lambda}$, decreases rapidly, and $\Omega_{\Lambda}$ soon dominates the evolution of the universe, whereby a de Sitter universe is resulted.

From the metrics of Eqs.(3.3) and (3.5), on the other hand, one may naively conclude that the radion in the 


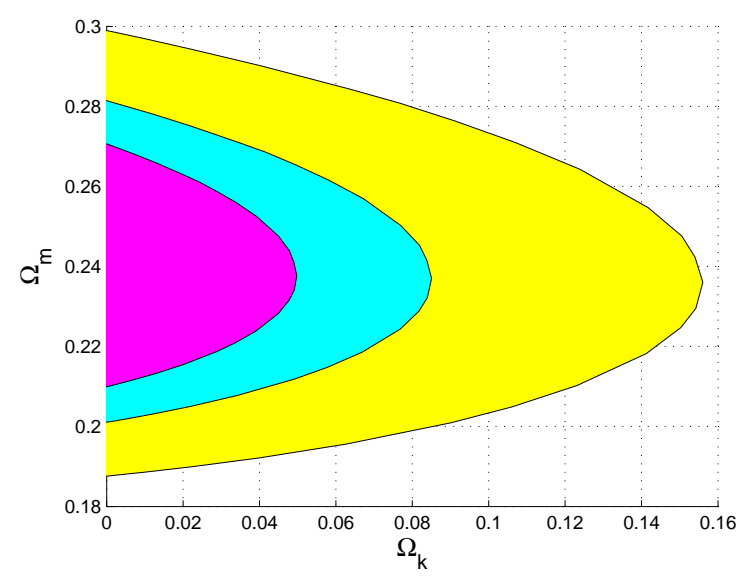

FIG. 3: The marginalized contour of $\Omega_{m}-\Omega_{k}$ for the potential given by Eq. (3.22) with $n=1$.

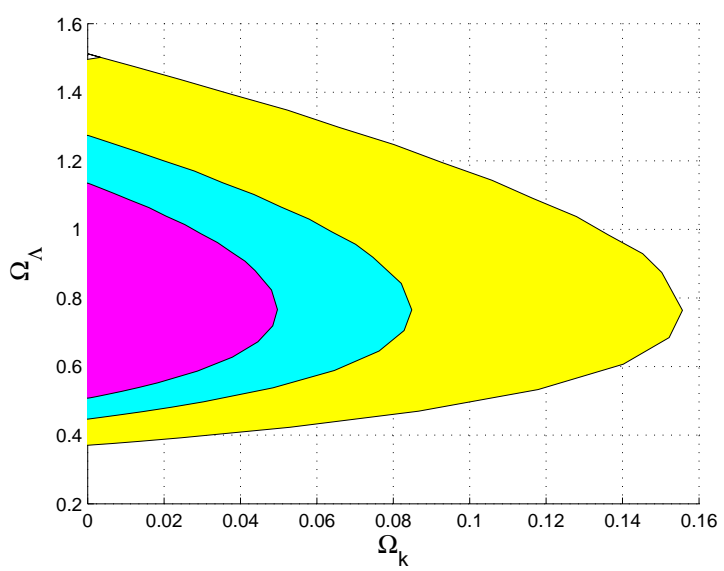

FIG. 4: The marginalized contour of $\Omega_{k}-\Omega_{\Lambda}$ for the potential given by Eq. (3.22) with $n=1$.

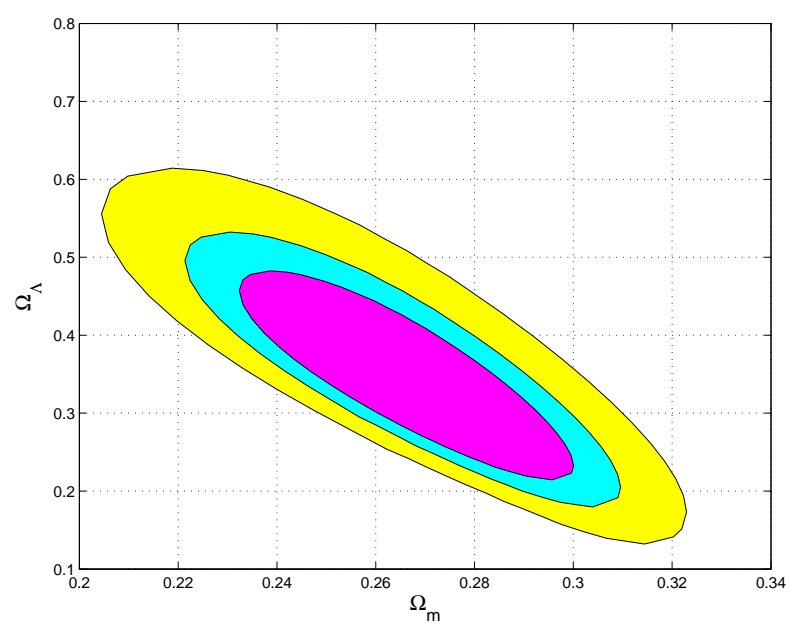

FIG. 5: The marginalized contour of $\Omega_{m}-\Omega_{\Lambda}$ for the potential given by Eq.(3.22) with $n=3.5$.

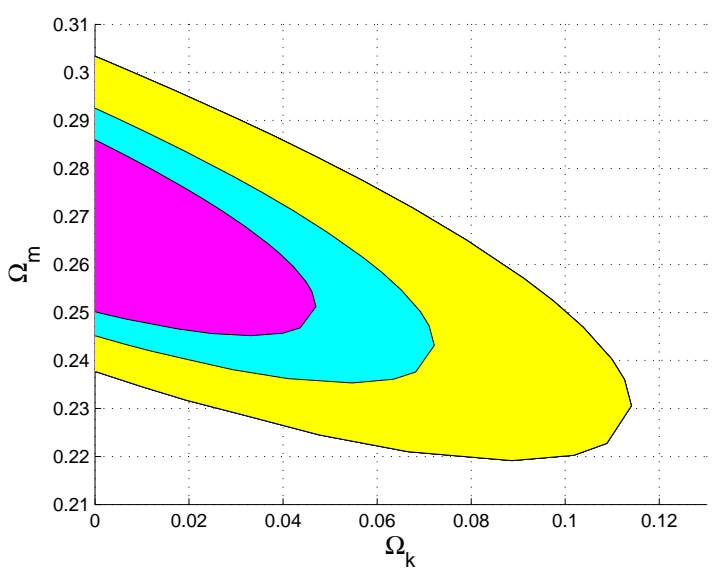

FIG. 6: The marginalized contour of $\Omega_{m}-\Omega_{k}$ for the potential given by Eq.(3.22) with $n=3.5$.

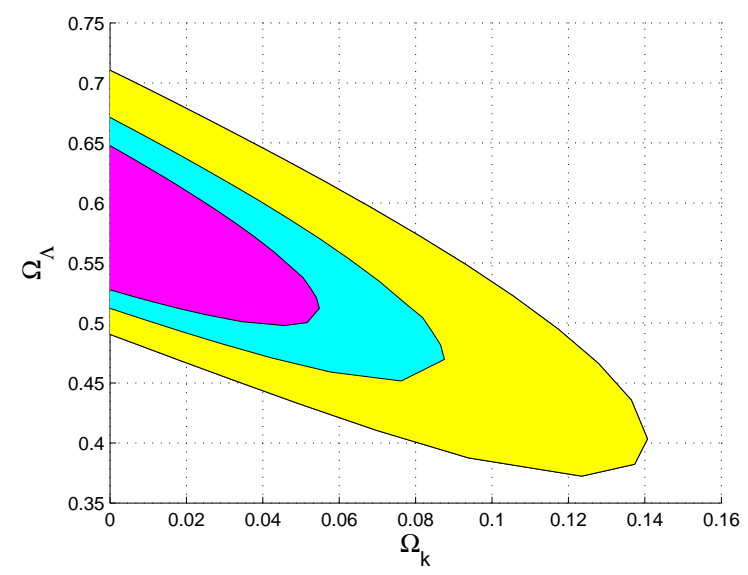

FIG. 7: The marginalized contour of $\Omega_{k}-\Omega_{\Lambda}$ for the potential given by Eq. (3.22) with $n=3.5$.

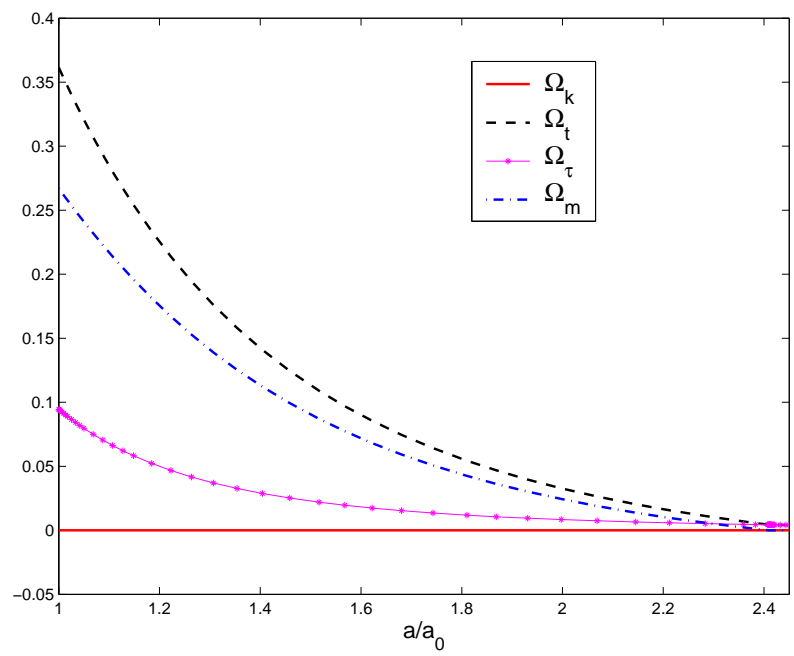

FIG. 8: The evolution of the matter components, $\Omega_{i}$ 's, for the potential given by Eq.(3.22) with $n=3.5$. 


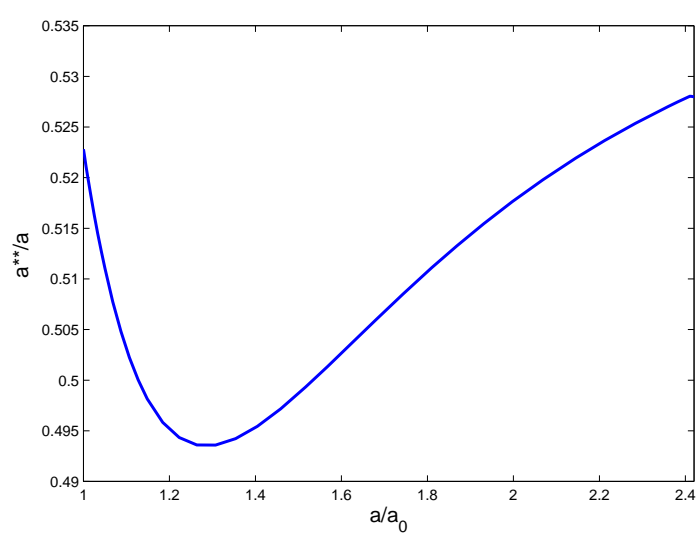

FIG. 9: The evolution of the acceleration $a^{* *} / a \equiv$ $\left(d^{2} a / d\left(H_{0} \tau\right)^{2}\right) / a$ for the potential given by Eq.(3.22) with $n=3.5$.

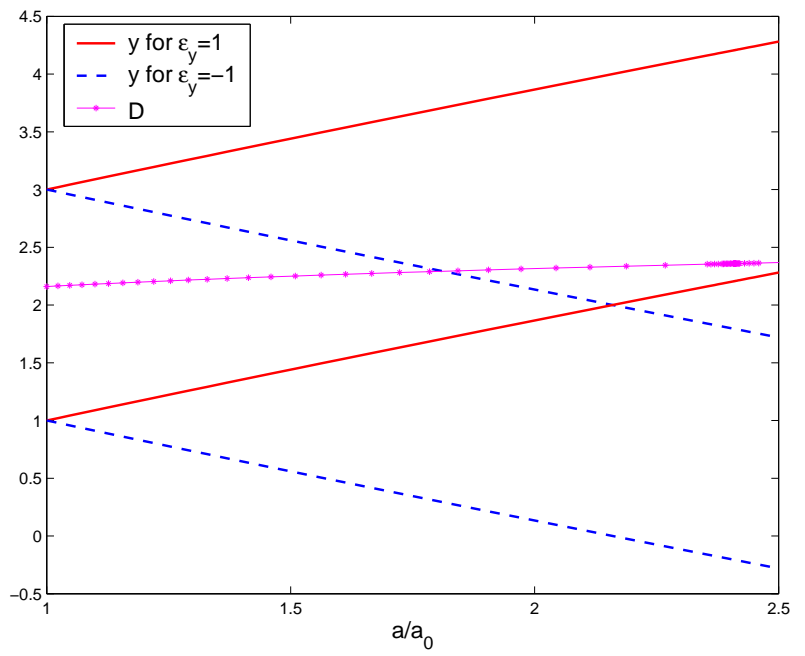

FIG. 10: The locations of the two branes $y_{I}(a)$, and the proper distance $\mathcal{D}$ between the two branes for the potential given by Eq. 3.22 with $n=3.5$. The initial conditions are chosen so that $y_{1}\left(a_{0}\right)=3$ and $y_{2}\left(a_{0}\right)=1$. The choice of $\epsilon_{y}=+1$ $\left(\epsilon_{y}=-1\right)$ corresponds to the case where the branes move towards the increasing (decreasing) direction of $y$.

present case is not stable, as the proper distance given by Eq.(2.55) seems either to increases to infinity (in the Einstein frame, given by Eq.(3.3) ) or to decreases to zero (in the string frame, given by Eq.(3.5)), as $t \rightarrow \infty$. A closer investigation shows that the problem is not as simple as it looks like. In particular, since $y_{I}=y_{I}\left(\tau_{I}\right)$, Eq. 2.55) makes sense only when the relation $\tau_{1}=\tau_{1}\left(\tau_{2}\right)$ is known. In the present case, we transform such a dependence to the expansion factor $a$, and plot it out in Fig. 10, together with $y_{I}(a)$, from which we can see clearly that the distance between the two branes remains almost constant. This indicates that the radion might be stable. Certainly, before a definitive conclusion is reached, more detailed investigations are needed.

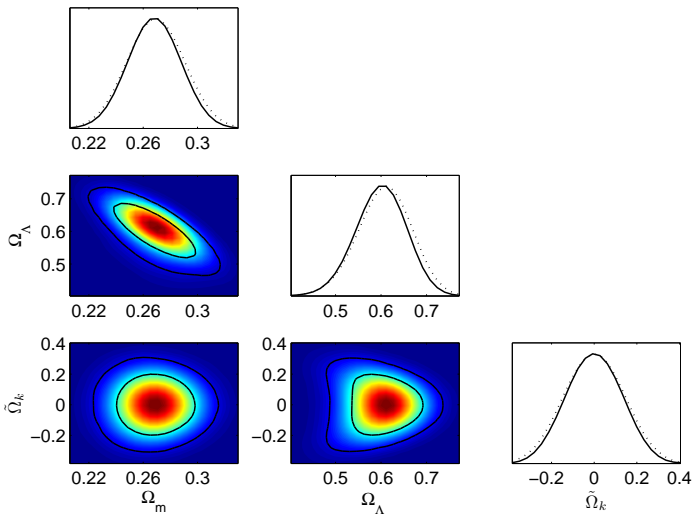

FIG. 11: The marginalized probabilities and contours for the potential given by Eq. (3.22) with $n=3.5$.

We also fit the above model with $n=3.5$ by using our Monte-Carlo Markov Chain (MCMC) code 25], based on the publicly available package COSMOMC [37], and find that the best fitting is $\Omega_{m}=0.27 \pm_{0.03}^{0.04}, \Omega_{\Lambda}=0.61 \pm_{0.10}^{0.09}$, and $\tilde{\Omega}_{k}=-0.0026 \pm{ }_{0.2396}^{0.2339}$ with $\chi^{2}=164.10$, where

$$
\Omega_{k} \equiv \tilde{\Omega}_{k}^{2}
$$

The corresponding marginalized probabilities and contours are given in Fig. 11] Clearly, these best fitting values are consistent with those obtained above by using our MINUIT code [24].

$$
\text { 2. } V_{4}^{(I)}=\lambda_{4}^{(I)}\left(\psi^{2}-v_{I}^{2}\right)^{2}
$$

To stabilize the radion, Goldberger and Wise proposed to choose the potential $V_{4}^{(I)}$ as 38],

$$
V_{4}^{(I)}(\phi, \psi)=\lambda_{4}^{(I)}\left(\psi^{2}-v_{I}^{2}\right)^{2},
$$

where $\lambda_{4}^{(I)}$ and $v_{I}^{2}(I=1,2)$ are constants. Then, we find that

$$
\Omega_{\tau}^{(I)}=\Omega_{\tau}^{(0, I)}\left(\left(\frac{3}{\sqrt{40}} \ln (a)\right)^{2}-v_{I}^{2}\right)^{2}
$$

where $\Omega_{\tau}^{(0, I)} \equiv \epsilon_{I} \lambda_{4}^{(I)} / \rho_{c r}$. Note that in writing the above expressions, without loss of any generality, we had set $\psi_{0}=0$. Then, the fitting parameters now can be taken as,

$$
\left\{\Omega_{\Lambda}, \Omega_{m}^{(0)}, \Omega_{k}^{(0)}, v_{I}\right\}
$$

Fitting the above model to the 182 gold supernova Ia data [22] and the BAO parameter from SDSS [23], we first study the dependence of $\chi^{2}$ on $v_{I}$. Table 1 shows such a dependence and the best fitting values of $\Omega_{i}$ 's for each given $v_{I}$. 


\begin{tabular}{|c|c|c|c|c|c|}
\hline$v_{I}$ & $\chi^{2}$ & $\Omega_{m}$ & $\tilde{\Omega}_{k}$ & $\Omega_{\Lambda}$ & $\Omega_{\Lambda}+\Omega_{\tau}$ \\
\hline 0.1 & 171.28 & $0.25 \pm_{0.04}^{0.03}$ & $-0.0009 \pm_{0.22}^{0.21}$ & $0.72 \pm_{0.05}^{0.05}$ & 0.73 \\
\hline 0.3 & 168.10 & $0.29 \pm_{0.05}^{0.05}$ & $-0.0006 \pm_{0.41}^{0.41}$ & $1.06 \pm_{0.17}^{0.15}$ & 0.47 \\
\hline 0.5 & 157.50 & $0.29 \pm_{0.04}^{0.04}$ & $-0.008 \pm_{0.44}^{0.46}$ & $1.28 \pm_{0.28}^{0.31}$ & 0.70 \\
\hline 1.0 & 156.69 & $0.29 \pm_{0.04}^{0.03}$ & $-0.002 \pm_{0.52}^{0.52}$ & $1.64 \pm_{0.48}^{0.71}$ & 0.64 \\
\hline 3.0 & 156.38 & $0.28 \pm_{0.04}^{0.03}$ & $-0.008 \pm_{0.56}^{0.53}$ & $1.93 \pm_{0.73}^{1.01}$ & 0.57 \\
\hline 10.0 & 166.35 & $0.28 \pm_{0.03}^{0.05}$ & $-0.002 \pm_{0.62}^{0.62}$ & $1.97 \pm_{0.74}^{2.17}$ & 0.56 \\
\hline
\end{tabular}

TABLE I: The best fitting values of $\Omega_{i}$ for a given $v_{I}$ of the potential given by Eq. 3.26).

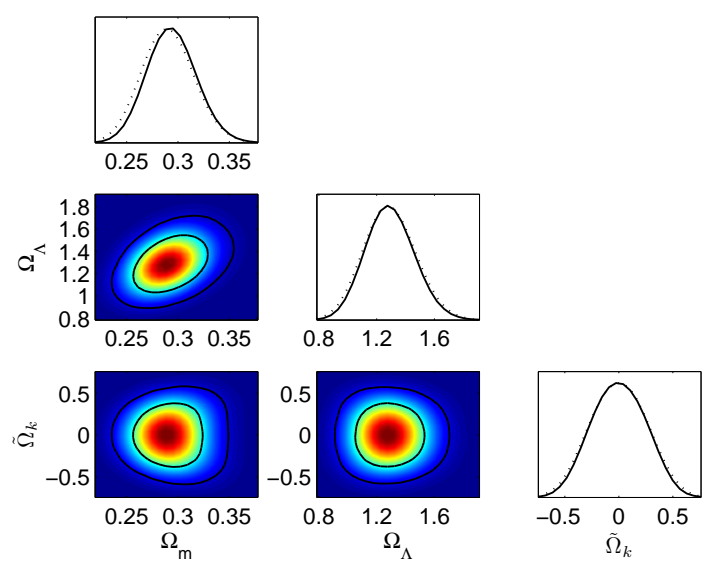

FIG. 12: The marginalized probabilities and contours for the potential given by Eq.(3.26) with $v_{I}=0.5$.

From the table we can see that $\chi^{2}$ decreases until $v_{I} \simeq 3.0$ and then starts to increase, as $v_{I}$ is continuously increasing. However, $\Omega_{\Lambda}$ and its uncertainty also increase as $v_{I}$ is increasing, while $\Omega_{m}$ and $\Omega_{k}$ remain almost the same. Since $\Omega_{\tau}$ acts as a varying cosmological constant, Table \ shows that the total effective cosmological constant $\Omega_{\Lambda}^{e f f} \equiv \Omega_{\Lambda}+\Omega_{\tau}$ is between 0.47 and 0.73 .

Fig. 12 shows the marginalized probabilities and contours for the potential given by Eq.(3.26) with $v_{I}=0.5$, and Fig. 13 shows the future evolution of the corresponding acceleration of the universe. From there we can see that the acceleration increases to a maximal value and then starts to decrease. As the time is continuously increasing, it will pass the zero point and then becomes negative. Thus, in the present model, the domination of the cosmological constant is only temporary. Due to the presence of the potential term, represented by $\Omega_{\tau}$, the universe will be in its decelerating expansion phase again in the future, whereby all problems connected with a far future de Sitter universe are resolved [20]. The effects of $\Omega_{\tau}$ can be seen clearly from Fig. 14, from which we can see that both $\Omega_{m}$ and $\Omega_{k}$ decrease rapidly, and soon $\Omega_{\tau}$ dominates the evolution of the universe.

These are the common features for any given value of $v_{I}$. Figs. 15] 16] and 17 show, respectively, the marginal-

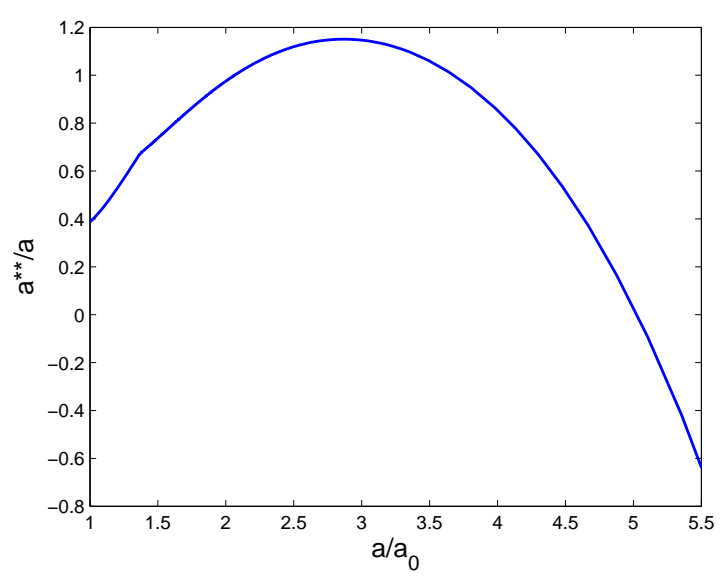

FIG. 13: The acceleration $a^{* *} / a$ for the potential given by Eq.(3.26) with $v_{I}=0.5$.

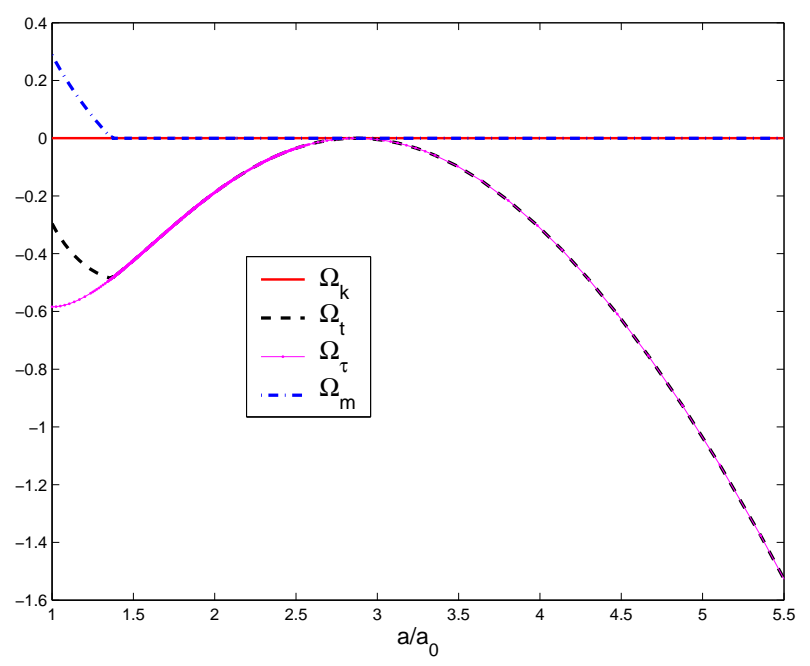

FIG. 14: The future evolution of $\Omega_{i}$ for the potential given by Eq.3.26 with $v_{I}=0.5$.

ized probabilities and contours, the future evolution of $a^{* *} / a$ and of $\Omega_{i}$ for $v_{I}=0.1$.

In addition, we also find that the proper distance between the two orbifold branes defined by Eq.(2.55) is not sensitive to the choice of $v_{I}$, and remains almost constant during the future evolution of the universe, as shown in Fig. 18. This also indicates that the radion might be stable in the present case, too.

\section{CONCLUSIONS AND REMARKS}

Recently, we studied the cosmological constant problem in the framework of both M-theory [17] and string theory [21] on $S^{1} / Z_{2}$, and showed that, among other things, the effective cosmological constant on the branes can be easily lowered to its current observational value using the ADD large extra dimension mechanism [19]. 

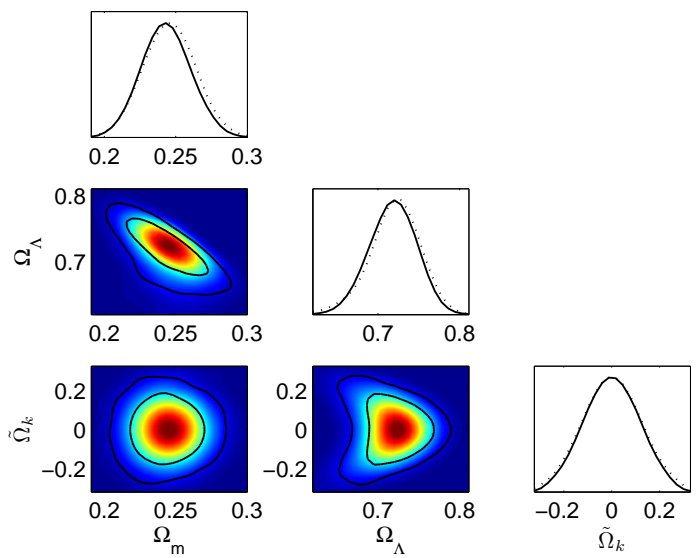

FIG. 15: The marginalized probabilities and contours for the potential given by Eq. (3.26) with $v_{I}=0.1$.

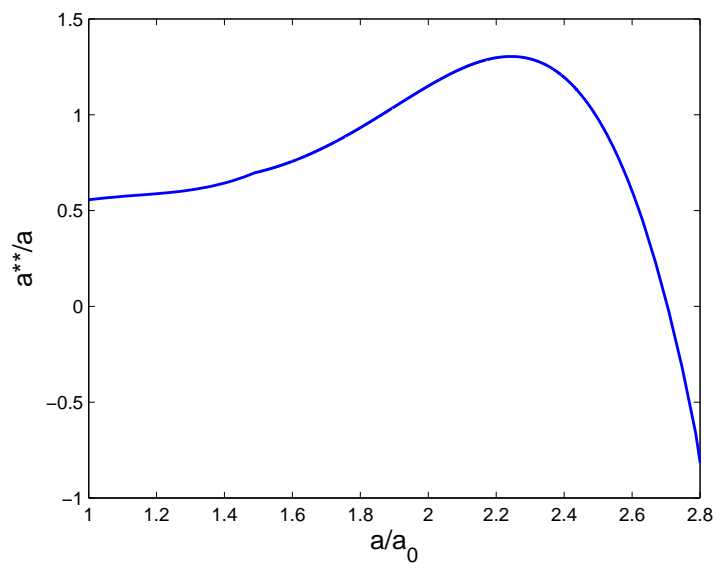

FIG. 16: The acceleration $a^{* *} / a$ for the potential given by Eq.(3.26) with $v_{I}=0.1$.

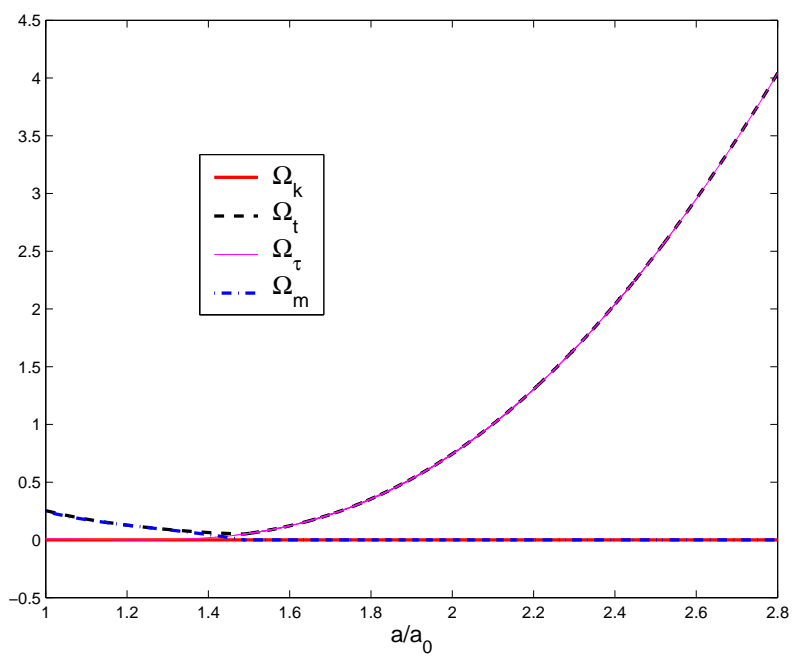

FIG. 17: The future evolution of $\Omega_{i}$ for the potential given by Eq.3.26 with $v_{I}=0.1$.

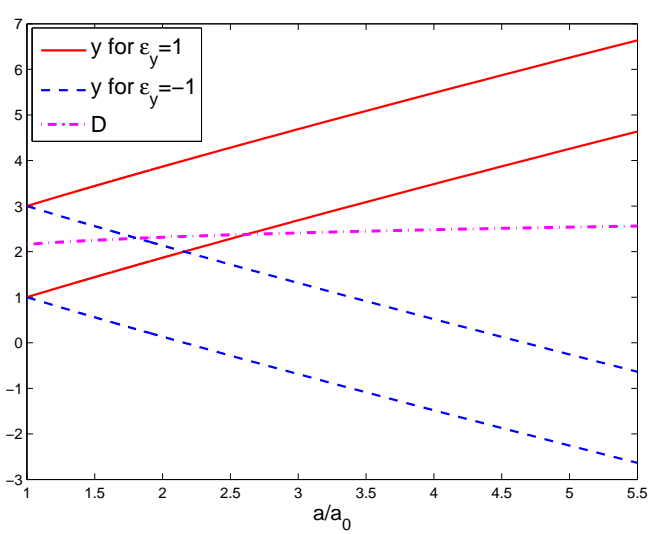

FIG. 18: The locations of the two branes, $y_{I}(a)$, and the proper distance, $\mathcal{D}$, between the two branes for the potential given by Eq.(3.26) with $v_{I}=0.5$. The initial conditions are chosen so that $y_{1}\left(a_{0}\right)=3$ and $y_{2}\left(a_{0}\right)=1$. The choice of $\epsilon_{y}=+1\left(\epsilon_{y}=-1\right)$ corresponds to the case where the branes move towards the increasing (decreasing) direction of $y$.

Thus, brany cosmology of string/M-Theory seems to have a built-in mechanism for solving both the cosmological constant and the hierarchy problems.

In this paper, we have studied a particular cosmological model in the framework of string theory on $S^{1} / Z_{2}$, developed in [21]. We have first solved the field equations in the bulk and then studied its local and global properties. In particular, we have found that the 10-dimensional bulk has a big-bang-like singularity at $t=0$.

After obtained explicitly the generalized Friedmann equations on each of the two branes for any radion potentials $V_{4}^{(I)}(I=1,2)$ of the branes, we have studied two different cases where $V_{4}^{(I)}=V_{(4)}^{0} \exp \left\{\frac{n}{2}\left(\frac{5}{\sqrt{6}} \phi+\sqrt{\frac{5}{2}} \psi\right)\right\}$ and $V_{4}^{(I)}=$ $\lambda_{4}^{(I)}\left(\psi^{2}-v_{I}^{2}\right)^{2}$, respectively. For each of these potentials, we have fit the corresponding models to the 182 gold supernova Ia data [22] and the BAO parameter from SDSS [23], and obtained the best fitting values of the parameters involved. To doubly check our numerical codes, we have used both of our MINUIT 24] and Monte-Carlo Markov Chain (MCMC) 25] codes, and gotten the same results within the allowed errors. With these best fitting values as the initial condition, we have integrated numerically the field equations on the branes, and found the future evolution of the universe. In particular, for $V_{4}^{(I)}=\lambda_{4}^{(I)}\left(\psi^{2}-v_{I}^{2}\right)^{2}$, we have found that the current acceleration of the universe driven by the effective cosmological constant is only temporary. Due to the effects of the potentials, the universe will be in its decelerating expansion phase again in the future. We have also studied the proper distance between the two orbifold branes, and found that it remains almost constant during the whole future evolution of the universe in all these models.

In the framework of orbifold branes, an important 
question is the radion stability. The considerations of the proper distance between the two orbifold branes indicate that in these cases the radion might be stable, although further studies are highly demanded. Recently, two of the authors (NOS \& AW) studied the problem in a static background with a four-dimensional Poincaré symmetry,

$$
d s_{5}^{2}=e^{2 \sigma(y)}\left(\eta_{\mu \nu} d x^{\mu} d x^{\nu}-d y^{2}\right),
$$

where

$$
\begin{aligned}
\sigma(y) & =\frac{1}{9} \ln \left|y+y_{0}\right| \\
\phi(y) & =-\frac{5}{\sqrt{54}} \ln \left|y+y_{0}\right|+\phi_{0}, \\
\psi(y) & =-\sqrt{\frac{5}{18}} \ln \left|y+y_{0}\right|+\psi_{0},
\end{aligned}
$$

and $y_{0}, \phi_{0}$ and $\psi_{0}$ are the integration constants with,

$$
\psi_{0}=\sqrt{\frac{2}{5}}\left[\ln \left(\frac{2}{9 V_{(5)}^{0}}\right)-\frac{5}{\sqrt{6}} \phi_{0}\right] .
$$

Following [39], we are currently investigating the radion stability, and wish to report our findings some where else soon.

\section{Acknowledgments}

The authors AW and QW would like to thank Dr. Yungui Gong for valuable discussions and suggestions. This work was initiated when the authors NOS and AW were visiting LERMA/CNRS-FRE, Paris. They would like to thank the Laboratory for hospitality. This work was partially supported by NSFC under grant No. 10775119 and No. 10703005 (AW \& QW).
[1] S. Weinberg, Rev. Mod. Phys. 61, 1 (1989); S.M. Carroll, arXiv:astro-ph/0004075; T. Padmanabhan, Phys. Rept. 380, 235 (2003); S. Nobbenhuis, arXiv:gr-qc/0411093 J. Polchinski, arXiv:hep-th/0603249 and J.M. Cline, arXiv:hep-th/0612129.

[2] A.G. Riess et al., Astron. J. 116, 1009 (1998); S. Perlmutter et al., Astrophys. J. 517, 565 (1999).

[3] A.G. Riess et al., Astrophys. J. 607, 665 (2004); P. Astier et al., Astron. and Astrophys. 447, 31 (2006); D.N. Spergel et al.,Astrophys. J. Supl. 170, 377 (2007) astro-ph/0603449; W.M. Wood-Vasey et al., astro-ph/0701041 and T.M. Davis et al., astro-ph/0701510

[4] S. Sullivan, A. Cooray, and D.E. Holz, JCAP, 09, 004 (2007) arXiv:0706.3730; A. Mantz, et al., Mon. Not. Roy. Astron. Soc. 387, 1179 (2008) arXiv:0709.4294; and J. Dunkley, et al., arXiv:0803.0586.

[5] A. Albrecht, et al, arXiv:astro-ph/0609591; J.A. Peacock, et al, arXiv:astro-ph/0610906

[6] L. Susskind, arXiv:hep-th/0302219

[7] R. Bousso and J. Polchinski, JHEP, 006, 006 (2000).

[8] P.K. Townsend and N.R. Wohlfarth, Phys. Rev. Lett. 91, 061302 (2003).

[9] G. W. Gibbons in Supersymmetry, Supergravity and Related Topics, edited by F. de Aguila, et al (Sigapore, World Scientific, 1985), p.124; and J.M. Maldacena and C. Nuñez, Int. J. Mod. Phys. A16, 822 (2001).

[10] N. Ohta, Phys. Rev. Lett. 91, 061303 (2003).

[11] N.R. Wohlfarth, Phys. Lett. B563, 1 (2003); and S. Roy, ibid., 567, 322 (2003).

[12] J.K. Webb, et al, Phys. Rev. Lett. 87, 091301 (2001); J.M. Cline and J. Vinet, Phys. Rev. D68, 025015 (2003); N. Ohta, Prog. Theor. Phys. 110, 269 (2003); Int. J. Mod. Phys. A20, 1 (2005); C.M. Chen, et al, JHEP, 10, 058 (2003); E. Bergshoeff, Class. Quantum Grav.
21, 1947 (2004); Y. Gong and A. Wang, ibid., 23, 3419 (2006); I.P. Neupane and D.L. Wiltshire, Phys. Rev. D72, 083509 (2005); Phys. Lett. B619, 201 (2005); K. Maeda and N. Ohta, ibid., B597, 400 (2004); Phys. Rev. D71, 063520 (2005); K. Akune, K. Maeda and N. Ohta, ibid., 103506 (2006) V. Baukh and A. Zhuk, ibid., 73, 104016 (2006); A. Krause, Phys. Rev. Lett. 98, 241601 (2007); I.P. Neupane, ibid., 98, 061301 (2007); and references therein.

[13] N. Arkani-Hamed, et al, Phys. Lett. B480, 193 (2000); and S. Kachru, M.B. Schulz, and E. Silverstein, Phys. Rev. D62, 045021 (2000).

[14] Y. Aghababaie, et al, Nucl. Phys. B680, 389 (2004); JHEP, 0309, 037 (2003); C.P. Burgess, Ann. Phys. 313, 283 (2004); AIP Conf. Proc. 743, 417 (2005); and C.P. Burgess , J. Matias, and F. Quevedo, Nucl. Phys. B706, 71 (2005).

[15] S. Forste, et al, Phys. Lett. B481, 360 (2000); JHEP, 0009, 034 (2000); C. Csaki, et al, Nucl. Phys. B604, 312 (2001); and J.M. Cline and H. Firouzjahi, Phys. Rev. D65, 043501 (2002).

[16] C.P. Burgess, arXiv:0708.0911.

[17] Y.-G. Gong, A. Wang, and Q. Wu, Phys. Lett. B663, 147 (2008) arXiv:0711.1597.

[18] H. Horava and E. Witten, Nucl. Phys. B460, 506 (1996); 475, 94 (1996).

[19] N. Arkani-Hamed, S. Dimopoulos and G. Dvali, Phys. Lett. B429, 263 (1998); Phys. Rev. D59, 086004 (1999); and I. Antoniadis, et al., Phys. Lett., B436, 257 (1998).

[20] W. Fischler, et al., JHEP, 07, 003 (2001); J.M. Cline, ibid., 08, 035 (2001); E. Halyo, ibid., 10, 025 (2001); S. Hellerman, ibid., 06, 003 (2003); L.M. Krauss and R.J. Scherrer, Gen. Relativ. Grav. 39, 1545 (2007) arXiv:0704.0221; and references therein.

[21] A. Wang and N.O. Santos, Phys. Lett. B, in press 
(2008) arXiv:0712.3938.

[22] A.G. Riess, et al, arXiv:astro-ph/0611572 (2006).

[23] D.J. Eisenstein et al, Astrophys. J. 633, 560 (2005).

[24] Q. Wu, Y.-G. Gong, A. Wang, and J.S. Alcaniz, Phys. Lett. B659, 34 (2008) arXiv:0705.1006; and Y.-G. Gong and A. Wang, Phys. Rev. D75, 043520 (2007) arXiv:astro-ph/0612196; ibid., D73, 083506 (2006) arXiv:astro-ph/0601453.

[25] Y.-G. Gong, Q. Wu, and A. Wang, Astrophys. J. 681, 27 (2008) arXiv:0708.1817.

[26] V. Sahni and Y.Shtanov, JCAP, 0311, 014 (2003) arXiv:astro-ph/0202346.

[27] G. R. Dvali, G. Gabadadze and M. Porrati, Phys. Lett. B484, 112 (2000); and C. Deffayet, ibid., 502, 199 (2001).

[28] J.E. Lidsey, D. Wands, and E.J. Copeland, Phys. Rept. 337, 343 (2000); and M. Gasperini, Elements of String Cosmology (Cambridge University Press, Cambridge, 2007).

[29] T. Battefeld and S. Watson, Rev. Mod. Phys. 78, 435 (2006).

[30] F. Leblond, R.C. Myers, and D.J. Winters, JHEP, 07, 031 (2001).

[31] A. Wang, R.-G. Cai, and N.O. Santos, Nucl. Phys. B797, 395 (2008) arXiv:astro-ph/0607371.

[32] T. Shiromizu, K.-I. Maeda, and M. Sasaki, Phys. Rev.
D62, 024012 (2000); A.N. Aliev and A.E. Gumrukcuoglu, Class. Quantum Grav. 21, 5081 (2004); and R.-G. Cai and L.-M. Cao, Nucl. Phys. B785, 135 (2007).

[33] C. Lanczos, Phys. Z. 23, 539 (1922); Ann. Phys. (Germany), 74, 518 (1924); and W. Israel, Nuovo Cimento B44, 1 (1967); (Errata) B48, 463 (1967).

[34] J.A. Wolf, Spaces of Constant Curvature, Fifth Edition (Publish or Perish, Inc., Wilmington, Delaware, U.S.A., 1984).

[35] I. Antoniadis, J. Phys. Conf. Ser. 8, 112 (2005); and V.H.S. Kumar and P.K. Suresh, arXiv:hep-th/0606194.

[36] J.C. Long et al., Nature (London), 421, 922 (2003); and D.J. Kapner, et al., Phys. Rev. Lett. 98, 021101 (2007).

[37] A. Lewis and S. Bridle, Phys. Rev. D66, 103511 (2002).

[38] W.D. Goldberger and M.B. Wise, Phys. Rev. Lett. 83, 4922 (1999).

[39] J. Garriga and T. Tanaka, Phys. Rev. Lett. 84, 2778 (2000); T. Tanaka and X. Montes, Nucl. Phys. B582, 259 (2000); O. DeWolfe, D.Z. freedman, S.S. Gubser, and A. Karch, Phys. Rev. D62, 046008 (2000); C. Csáki, M.L. Graesser, and G.D. Kribs, ibid., 63, 065002 (2001); J. Lesgourgues and L. Sorbo, ibid., 69, 084010 (2004); and D. Konikowska, M. Olechowski, and M.G. Schmidt, ibid., 73, 105018 (2006). 